\title{
Institutional Obstacles to Ethnographic Observation in Engineering Industry
}

\section{Prof. Reed Stevens, Northwestern University}

Reed Stevens is a Professor of Learning Sciences at Northwestern University. He holds a B.A. in Mathematics from Pomona College and $\mathrm{PhD}$ in Cognition and Development from the University of California, Berkeley. Professor Stevens began his professional career as a mathematics teacher. For the past two decades, he has studied STEM learning both in and out of school. His research seeks to understand how and when learning environments are productive for people and to translate those findings into practical use in the design and resdesign of learning environments. In recent years and in collaboration with colleagues at Northwestern, he created FUSE Studios to reimagine STEAM education around the values of innovation, making, and collaboration (https://www.fusestudio.net/). Since 2012, FUSE has been implemented in more than 135 school and community sites around the US and will soon begin in Helsinki, Finland. Stevens co-led two large National Science Foundation (NSF) research centers-the Center for the Advancement of Engineering Education (CAEE) and the Learning in Informal and Formal Environments Center (LIFE) - and has received numerous research grants from NSF and various private foundations. Articles of his relevant to the engineering education research community include "Becoming an engineer: Toward a three dimensional view of engineering learning" (2008) in the Journal of Engineering Education and "Professional engineering work" (2014) in the Cambridge handbook of engineering education research.

\section{Dr. Alexandra Vinson, Northwestern University}

Alexandra H. Vinson is a Postdoctoral Fellow in the School of Education and Social Policy at Northwestern University. She received her Ph.D. in Sociology \& Science Studies from the University of California, San Diego. Her research interests include professional education and enculturation in medicine and STEM fields. 


\section{Institutional Obstacles to Ethnographic Observation in Engineering Industry}

\section{Introduction}

One style of research in engineering education and other social science disciplines is ethnographic fieldwork. Ethnographic fieldwork involves researchers identifying contexts of everyday activity to study, negotiating access to those contexts, and then conducting the fieldwork. Fieldwork data collection typically involves some combination of observation, making recordings of everyday activity, and interviewing.

While researchers can learn a great deal from interviews, for more than a half-century social science researchers have also stressed the primary importance of direct observation. In a classic analysis comparing what can be learned from interviewing vs. participant observation - "that method in which the observer participates in the daily life of the people under study," Becker and Geer argued that conducting only interviews, without complementary observations about what is being discussed in interviews, has a number of limitations. The challenges they identified of conducting research with only interviews included, "learning the native language, or the problem of the degree to which the interviewer really understands what is said to him; matters interviewees are unable or unwilling to talk about; and getting information on matters people see through distorting lenses." 1

What Becker \& Geer mean by the 'learning the native language' is exemplified by a story of how Becker learned the meaning of the word "crock" in his participant observations among medical students. ${ }^{2}$ The learning the native language issue in engineering education can be illustrated with a question about the meaning of a term like 'teamwork.' Though nearly every engineer we might think to interview would likely highlight the importance of 'teamwork' to engineering practice, our prior work suggests that this common term (and thereby any common term) can hide a great deal of diversity in the daily lived practice of "teamwork." Only by direct observation can we see what teamwork looks like in different organizational contexts and see whether what one engineer counts as teamwork is similar, or not, to what another counts. Some engineers might count as teamwork only close face to face ongoing collaborative work while others might count more loosely coupled geographically distributed work.

We offer this example in the context of a NSF funded research project we have been conducting for a couple years, and which has as its focus what new engineers learn on the job as they are first entering the professional work setting. A major part of this study involves trying to understand what these very early career engineers learn anew in their first professional positions and how it relates to what they learned in their undergraduate engineering educations. Elsewhere we articulated multiple reasons why research that produces concrete images of professional work is important for engineering education. ${ }^{3}$ We were convinced, as were NSF panel reviewers, that it was important to fill a visible gap in the research literature on the learning and work practices of new engineers. And we found further motivation for the study from arguments in important consensus documents in engineering education. As stated in an authoritative consensus report on engineering education from the National Academy of Engineering, there is a clear "disconnect 
between engineers in practice and engineers in academe" and that "the great majority of engineering faculty, for example, have no industry experience. Industry representatives point to this disconnect as the reason that engineering students are not adequately prepared, in their view, to enter today's workforce" (italics added). "This is a situation that is hardly unique to engineering and concerns about gaps and mismatches between university education and professional practice are common in many fields including K-12 teaching, medicine, and law.

Because of perspectives like this from 'industry representatives,' we were hopeful that we would find willing research partners in industry who saw the value of this research for, if not directly closing the gaps between university education and professional practice, at least getting a clearer, empirically grounded understanding of these gaps. We and our research collaborators at another university went into this study with combined decades of ethnographic fieldwork experience in other professional engineering settings and related professional settings, ${ }^{5,6}$ as well as other challenging ethnographic field sites like family homes. ${ }^{7,8}$ Because of these prior fieldwork experiences, we were - in addition to being hopeful — also realistic that negotiating access settings would present challenges because every new setting presents its own challenges.

However, we had no reason to see these as insurmountable challenges. We were also as sensitive as possible in our research design; we planned what we call a "sliding scale" approach to each particular focal research participant (i.e. new engineer). Depending on how she or he felt about the research, we planned to vary the intensity of our field data collection, with an explicit plan to defer to more conservative data collection approaches if requested. As well, we went into the study knowing that many firms might be concerned about the protection of their intellectual property, so we stated in our grant proposal and have made good on in practice, a volunteered willingness to sign any and all non-disclosure agreements from professional engineering firms. Finally, we recognized the importance of social networks as we began this study, so we secured a letter of cooperation from university leadership at Large Private University (LP) in charge of relationships with engineering firms (e.g. job fairs, job placements, coop and internship positions); these leaders have long standing relationships with engineering workplaces and have successfully helped us make initial appeals to a wide range of engineering workplaces.

As is likely anticipated by readers at this point, negotiating access to engineering workplaces to conduct our research on learning among new engineers has been a far greater challenge than expected. In what follows, we describe these challenges and attempt to put the difficulties into a broader context, both with respect to engineering as a profession and in terms of social science research that seeks to conduct direct, observational field research in certain kinds of settings.

\section{Perspectives on gaining access in ethnographic investigations}

The published texts in fields like sociology and anthropology about negotiating access to field sites is surprisingly sparse; a couple decades ago sociologist Thomas wrote that there is "a real paucity of literature available on how sociologists study elites, especially corporate elites." And despite an arguable increase in the importance of elites, technoscientific or otherwise, in society, this 'paucity' seems still to be the current state of affairs. ${ }^{10}$ A classic statement on this issue comes from anthropologist Laura Nader, who wrote about the importance of, but challenges of, "studying up," by which she meant studying people of equal or higher social or financial status than we researchers. She noted that at the time that there was "comparatively very little field 
research on the middle classes and very little first-hand work on the upper classes,"11 with most anthropological fieldwork at the time directed to studying the "powerless" rather than the "powerful," the "culture of poverty" rather than "the culture of affluence." Nader went on to argue that these asymmetries have consequences that are "serious in terms of developing adequate theory and description" (p.291). About anthropologists studying the powerful, Nader enumerates some of the different ways that powerful people are seemingly out of reach to research: "They don't want to be studied; it is dangerous to study the powerful; they are busy people; they are not all in one place, and so on" but she then goes on to say, "in view of our [i.e. anthropologists'] successes among peoples of the world that have been incredibly hostile, it is rather surprising anthropologists could be so timid at home" (p.302).

With the development of Science and Technology Studies (STS), elites in technoscientific fields have been studied with ethnographic field methods. A classic early study, Latour and Woolgar's Laboratory Life, ${ }^{12}$ reported on fieldwork conducted among scientists in an elite biology research organization, employing the trope that they were treating the scientists as "natives" the way traditional anthropology had treated indigenous or third world peoples. One of Latour's early synthetic accounts of the STS perspective had as its subtitle "How to Follow Scientists and Engineers Through Society."13 However, for the most part, it has mostly been scientists that have been followed through society. ${ }^{3,14}$ As a result, distinct challenges involved in conducting fieldwork with engineers as compared to scientists, have not sufficiently been confronted to date. Even though the ethnographic representations of scientific work from STS have been controversial, ${ }^{15}$ natural scientists have plausibly allowed themselves to be research subjects because they ultimately share with ethnographic social scientists a kind of "basic research" ethos - that if something is important and unstudied, or understudied, it is important for that research to proceed. The open question - if this is indeed why natural scientists are regularly willing to participate in ethnographic research - is: do engineers share this ethos and, more generally, what are their incentives and disincentives to participate in the kind of ethnographic field research we are describing here?

A recent paper on the issue of securing access to "secretive and guarded organizations" raises a number of points potentially germane to what we have found in our own multi-year attempt to negotiate access. Monahan and Fisher (2015) say that "individuals in positions of relative power may be more likely to refuse to participate in research studies," which is a version of Nader's view of the dilemmas of "studying up."10 Furthermore, these authors argue that "organizations typically have a range of gatekeepers [...] whose job is not just to filter outside requests but to respond to them, making it difficult for researchers to assert that their inquiries were not taken seriously."10

\section{Our research project}

Our project, called Learning Ethnographies of New Engineers, is a NSF funded collaboration between researchers at Northwestern University and researchers at the University of Colorado, Boulder. The core team includes two tenure line faculty, who serve as PI and co-PI, two postdoctoral scholars, and several graduate students. The research experiences we describe in this paper are drawn only from the team at Northwestern, though the Boulder team has informally reported very similar challenges, though perhaps for different reasons than we enumerate in what 
follows. Two post-doctoral scholars have been employed at Northwestern, one who participated in the project from May of 2014 to December 2014, at which time the first post-doctoral scholar left the project for personal reasons. In the time that this post-doctoral scholar worked with the project, his primary goal was the recruitment of research participants to the study. Despite making a dozen or initial contacts with 'new engineers' and gaining university approved IRB consent from eight of these participants, this post-doctoral scholar was unable to negotiate access to even a single engineering firm for fieldwork, though about half of these potential participants did agree to be interviewed outside the workplace. The most common reason offered for why these new engineers, who had given their consent to participate, could go no further is that their employers would not allow it.

A second post-doctoral scholar joined the project in May of 2015 (this paper's second author) and has worked tirelessly to recruit participants. During this period of recruitment, we made changes to our approach based on the prior phase. In this phase, we have typically sought to use our network connections to approach managers (or even heads of firms) first to determine whether they will allow our participation and only then to request informed consent from the new engineers. Despite this well justified change, the outcome thus far has largely been the same as during the first recruiting phase. New engineers are almost always willing to be interviewed outside of the workplace, but with only three exceptions, we have not secured access for fieldwork with those new engineers in their workplaces, despite 39 solid initial contacts and two additional firms that were (at the time of this paper) informally committed. These experiences have led us to carefully document and reflect on our recruitment experiences and in what follows we present data and analysis of these experiences. In turn, we speculate about the broader context that may be generating the unusual difficulties we have faced in securing fieldwork participation.

\section{Encountering difficulties in mediated recruitment}

In the first phase of research, we employed a direct recruitment strategy, which involved a researcher directly contacting potential study participants (i.e. new engineers) and asking them to be involved. While this process yielded eight engineers who consented to be part of the study, none ultimately were enrolled in the study because subsequently, their employers disallowed their participation for one reason or another. Following this outcome, in the second recruitment phase, we decided to add another strategy to our recruitment efforts, what we are calling a mediated recruitment strategy, in which we initially approached supervisory representatives in engineering organizations, established their initial interest, and subsequently sought to work within their organizational processes to ultimately invite a new engineer to participate, having cleared all the organizational hurdles.

Figure 1 (below) provides a synoptic image of the different points along the mediated recruitment 'gauntlet.' We have come to call this 'the gauntlet' because (as of the drafting of this manuscript) only three engineers have emerged as confirmed candidates for fieldwork out of 39 firms contacted or that expressed initial interest in having one or more of their employees participate. As this diagram illustrates, there are significant layers of 'gatekeepers' as Monahan and Fisher ${ }^{10}$ describe them. 


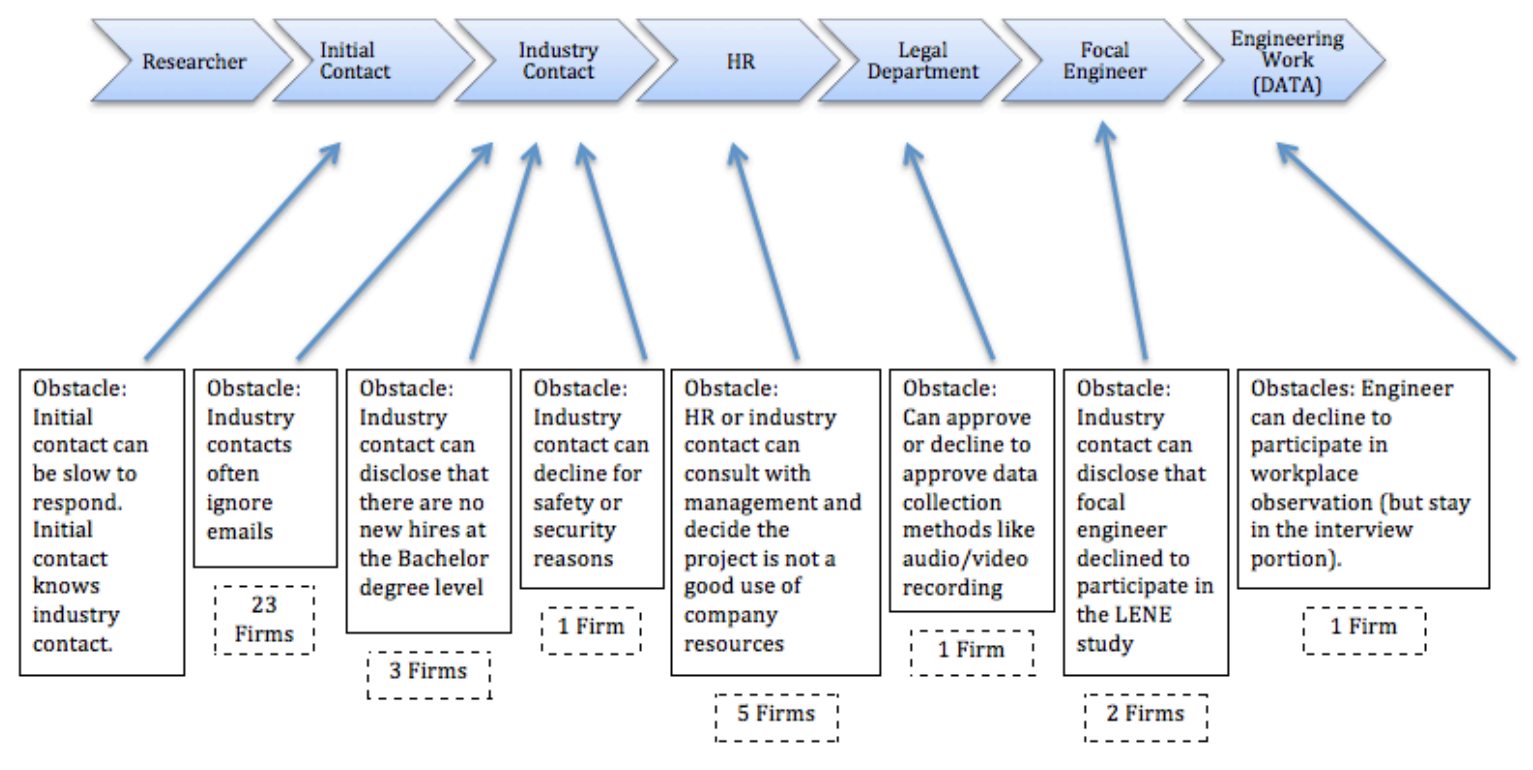

Figure 1. Mediated recruitment 'gauntlet'.

Next, we provide some specific details about the different reasons for declining that we have been given and the sources of those reasons (all names of people and organizations are pseudonyms). Among the reasons that organizational representatives gave for why their engineers could not participate were safety and security. As one industry representative explained, "much of our time is spent in a biohazard laboratory. In order to allow contractors or visitors into those areas, our facility requires proof of both training and certain immunizations for those individuals." This industry contact continued, "our group is the pipeline filler for the entire [specific category of business deleted] segment of the business. For this reason we are very sensitive about the intellectual property generated, discussed, and advanced on a daily basis. I understand that you have precautions in place to help ensure that our sensitive information is not shared, but we try to limit the exposure of our IP as much as possible."

Industry contacts also did not always have new hires on hand, sometimes due to hiring freezes, off times in the job cycle, or because the firm only hires more advanced engineers. One industry contact explained, "Sorry for the delay in response. Unfortunately with our current staff, and the planned additions in the future, I don't have any candidates for this program at this time. Likely the earliest this could occur is at the end of the school year. I will try to keep you posted on any potential new engineering hires. I will also talk more with my superiors to determine if something like this would be acceptable at our facility. Good luck with the study."

Another common reason for declining reported to us is that industry contacts, often in consultation with other management or executive level personnel, decide that participating is not a good use of company resources. For example, one industry contact politely declined, citing the 
constraints of organizational change: "I am going to pass at this time. We are in transition as [pharmaceutical company] was just purchased by [larger pharmaceutical company] and the close is expected in the next month or so. While our work continues and we are giving our students a top notch education, there are many areas that are shifting or will shift which does not lend itself to your research at this time."

On the few occasions where we have 'run the gauntlet' and we were cleared to have a direct request made to a new engineer, on two occasions we were told that the engineer herself or himself had declined, even after we had been informed of their initial potential interest in participating. In these cases, we did not have the opportunity to discuss the study directly with the new engineer; whatever she or he learned about our study, beyond any of our written materials, came from interactions with members of her or his employing organization. And it was these same organizational representatives who delivered the news that the new engineers had declined. We have no reason to be skeptical about these reports, but on the other hand, as Monahan and Fisher argue, it is difficult for us as researchers to determine how seriously our inquires were taken or how the study was framed, because we had no direct access or participation in the interactions with the would-be participants.

\section{Discussion}

We began this paper with statements from important consensus documents in engineering education like Educating the Engineer of 2020 that stated that "industry representatives" saw the "disconnect" between the academy and industry, "as the reason that engineering students are not adequately prepared, in their view, to enter today's workforce."4 Our strategy for addressing this "disconnect" is to conduct research as engineering students move into their first jobs. The animating idea behind this strategy is that by following the daily routines and experiences of new engineers on the job we will see what they have learned previously that they readily adapt to their current work contexts and, on the other hand, what is effectively 'new learning.' As researchers, we are agnostic on the current extent of the disconnect of 'preparation'; this is why set out to conduct the study in the first place: to replace uncertainty and an absence of empirical research with rich, grounded 'learning ethnographies of new engineers.' As educators hoping to contribute to the conversation about the future of engineering education, we feel strongly that whatever we learn can help bridge the gap and open up new conversations between the academy and industry.

There are many sound and understandable reasons we have been given for why participation in our study is declined. However, critical reflection has led us to one issue in particular that we wish to highlight. We have been struck in our recruiting interactions and in our broader explorations of engineering work, at this cultural moment, by how fully engineering organizations understand themselves almost exclusively as businesses. We were not naïve to this framing, of course, but we did hope and expect that engineering organizations would recognize the public research and educational mission of our study and balance it accordingly with the potential effects that our presence would have on their 'bottom line.' Based on our prior field research experience, we believe and have sought to be convincing that we would be as unobtrusive as possible. We also believe based on our past work and similar work by others in this tradition that real insights can be gained from our style of observational field research. Because 'industry representatives' themselves have highlighted the disconnect between the 
academy and industry, it seemed that more of them might open up opportunities for us to learn about the extent and particular qualities of the disconnect between learning in the university and learning in the early career workplace.

To do so however seems to require a degree of commitment to seeing engineering as a progressive human endeavor, above and beyond its obvious standing as a for-profit business enterprise. A larger vision common to many consensus documents in engineering education aligns engineering with all great professional traditions, in arguing that it also seeks to improve human lives. And engineering seems to play a particularly central role improving human lives in a world that faces increasingly complex sociotechnical problems. This is a view of engineering that we affirm, that it can be both profitable business and an agent of positive social change, just as we see the work of other professionals like doctors, architects, and teachers. We remain committed to the idea that our study can make an important contribution, and we hope, soon, that some 'industry representatives' come to share that view and will clear some space for us. In exchange, we promise to do our best to be as undisruptive as possible and likewise to aspire to 'add value,' if not in the very near term to an organization's bottom line, but in a reasonable middle term, to the important conversation that involves how our society examines and renegotiates the relationship between a college education and professional work.

\section{Acknowledgements}

This research was supported by a grant from the National Science Foundation (\#1252372).

\section{Bibliography}

[1] Becker, H. \& Blanche Geer (1957). "Participant observation and interviewing: A comparison.” Human Organization, 16(3): 28-32.

[2] Becker, H. (1993). How I learned what a crock was. Journal of Contemporary Ethnography, 22:28-35.

[3] Stevens, R. Johri, A., O’Connor, K. (2014). Professional Engineering Work. Johri, A., \& Olds, B., (Eds). Cambridge Handbook of Engineering Education Research.

[4] National Academy of Engineering of the National Academies (2005). Educating the Engineer of 2020: Adapting Engineering Education to the New Century. Washington D.C.: National Academies Press.

[5] Stevens, R. (2000). "Divisions of labor in school and in the workplace: Comparing computer and papersupported activities across settings." Journal of the Learning Sciences, 9(4), 373-401.

[6] Hall, R. Stevens, R. \& Torralba, A. (2003). "Disrupting representational infrastructure in conversations across disciplines." Mind, Culture, \& Activity, 9(3), 179-210.

[7] Stevens, R., Satwicz, T., \& McCarthy, L. (2008). “In game, In room, In world: Reconnecting video game play to the rest of kids' lives." The Ecology of Games. Salen, K. (Ed.), Cambridge: MIT Press.

[8] Stevens, R. \& Goodwin, M. (forthcoming). "Learning in Family Environments." Handbook for the Cultural Foundations of Learning. London: Routledge. 
[9] Thomas, R.J. (1993). “Interviewing Important People in Big Companies.” Journal of Contemporary Ethnography, 22(1):80-96.

[10] Monahan, T. \& Jill A. Fisher (2015). "Strategies for Obtaining Access to Secretive or Guarded Organizations." Journal of Contemporary Ethnography, 44(6):709-736.

[11] Nader, Laura 1974(1969) Up the Anthropologist-Perspectives Gained From Studying Up. In Dell Hymes (ed.) Reinventing Anthropology, New York: Vintage Books, pp. 284-311.

[12] Latour, B \& Steven Woolgar (1979) Laboratory Life: the construction of scientific facts. Princeton, NJ: Princeton University Press.

[13] Latour, B. (1987) Science in Action: How to follow scientists and engineers through society. Cambridge, MA: Harvard University Press.

[14] Downey, G., A. Donovan, \& T.J. Elliott (1989). "The invisible engineer: How engineering ceased to be a problem in science and technology studies." Knowledge \& Society, 8:189-216.

[15] Parsons, Keith (ed.) (2003). The Science Wars: Debating Scientific Knowledge and Technology, Prometheus Books, Amherst, NY USA. 